\title{
ACCOUPLEMENT ET FÉCONDATION DU FASCIOLA HEPATICA
}

\author{
Par N. LEON
}

La manière dont s'eflectue l'accouplement et la fécondation du Fasciola hepatica a donné lieu, comme pour le Diphyllobothrium latum, à plusieurs hypothèses :

$1^{\circ}$ Certains auteurs partagent l'avis de Stieda, à savoir que l'accouplement se fait par le canal de Laurer, qui représenterait le vagin. Dans ce cas, l'individu qui joue le rôle du mâle se placerait sur le dos de l'autre individu.

$2^{\circ}$ Une deuxième hypothèse est celle de la "réciprocité ". Deux individus s'accouplent ventre à ventre, à la façon des gastéropodes, chacun des deux fonctionnant à la fois comme mâle et comme femelle. La partie terminale de l'utérus sert de vagin.

$3^{\circ}$ Quelques auteurs émettent l'hypothèse de l'autocopulation, c'est-à-dire l'introduction du cirre dans la partie terminale de l'utérus, où le cirre déverserait la liqueur spermatique.

$4^{\circ}$ I'ne dernière hypothèse est celle de l'autofécondation sans copulation. D'après cette hypothèse, le cloaque génital se fermerait par la contraction de son système musculaire, et le sperme, déversé dans la poche cloacale, par le canal éjaculateur, passerait facilement dans le vagín et, de là, dans l'utérus.

De toutes ces hypothèses, celle de l'accouplement par utilisation du canal de Laurer comme vagin est la seule qui ait été abandonnée.

Restent les trois autres hypothèses où se sont arrêtés les auteurs: l'accouplement réciproque, l'autocopulation et l'autofécondation.

La plus probable de ces hypothèses, celle à laquelle s'est également arrêté Macé (1882), est celle d'une fécondation directe, sans copulation. " On trouve, en eflet, dit Macé, souvent des douves dont le cloaque sexuel est complètement fermé par la contraction des fibres musculaires de ses parois; chez certains de ces individus on peut parfois reconnaître la présence, dans la cavité ainsi formée, d'une masse analogue à celle que l'on trouve dans la vésicule séminale, des filaments spermatiques entourés d'un produit d'addition finement granuleux. On peut donc admettre que le sperme, poussé par les contractions des fortes parois musculaires du canal éjaculateur, ou par l'orifice màle, pénètre dans l'espèce de canal formé par

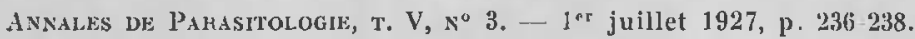


le rapprochement des hords du cloaque sexuel et parvient jusqu'à l'orifice femelle situé à la partie déclive de la cupule. Il progresse ensuite peu à peu à travers les sinuosités de l'oviducte et arrive jusqu'au commencement de ce conduit évacuateur, à l'endroit ai les œufs, ne possédant pas encore de coquille, sont tout prêts à subir son influence fécondante."

Nous n'objectons rien à ce mode de fécondation directe sans accouplement, car ce fait se produit dans la nature, mais nous n'excluons pas l'accouplement réciproque.

Schaeffer (1879) et Goze (1787) émettent l'avis que les Fasciola heprticu procèdent par accouplements réciproques: "dicht an

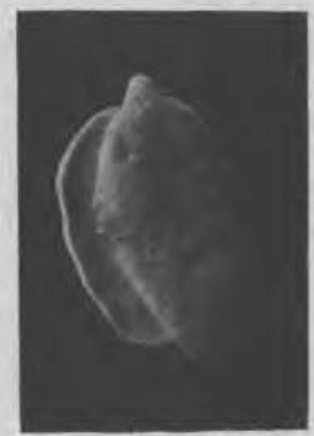

Fig. - Deux Fasciola hepalica au moment de l'accouplement.

einander Klebend in den Lebergängen so zu finden, dass das mänliche, wie ein Posthörnchen gekrümmte Glied des einem in dem weiblichen eines anderen, und umgekehrt steckten.

Aucun de ces auteurs n'a cependant eu sous les yeux un semblable accouplement.

Nous profitons de ce qu'il nous a été donné de trouver deux Fasciola au moment de leur copulation pour soutenir que l'accouplement s'opère selon l'hypothèse de Schaeffer et Goze.

On pourra écrire tant que l'on voudra au sujet de la copulation réciproque des Fasciola hepatica, aucun argument ne vaudra celui que nous offre la photographie ci-jointe (fig.).

Cette photographie nous montre deux Fascioln hepatica accolées par leur face ventrale. Le cirre de la Fasciola supérieure est introduit dans le canal vaginal de la Fasciola inférieure, alors que le cirre de celle qui est au-dessous est introduit dans le canal vaginal de celle qui est au-dessus. 
Lorsque nous avons séparé ces vers, le cirre de la Fasciola supérieure s'est rompu et est demeuré dans le vagin de la Fasciola inférieure ; quant au cirre de cette dernière, il présentait, en sortant du canal vaginal, une dimension très appréciable.

Macé a combattu l'hypothèse de l'accouplement réciproque à cause de l'énorme disproportion qui existe entre le cirre et le conduit qui doit le recevoir. Nous croyons qu'au moment de la copulation, lorsque le cirre s'introduit dans l'orifice vaginal, cet organe est très petit, mais qu'il grossit peu à peu, jusqu’à devenir énorme, lorsqu'il s'y amasse une grande quantité de sperme, provoquant, pour ainsi dire, une sorte d'érection.

\section{Bibliographie}

Baaun (....). - Broun's Klassen und Orduungen des Tierreichs, Vermes. Viertè Band., 1879-1893.

Goeze (I. A. E.). - Versuch einer Naturgeschichle der Eingeweidewürmer Thierischer Körper. Leipzig, 1787.

Mać́ (E.). - Recherches anatomiques sur la grande douve du foie. Nancy, 1882. Schăefrer. - Cité par Braun.

Laboraloire de Parasilologie de la Facullé de médecine de Jassy (Roumanie). 